\title{
Synergetic Effect of Tetraethylammonium Bromide Addition on the Morphology Evolution and Enhanced Photoluminescence of Rare-Earth Metal-Organic Frameworks
}

Jiaqiang Liu ${ }^{\dagger}$, Xianliang $\mathrm{Li}^{\star}$, Yide $\mathrm{Han}^{\dagger}$, Junbiao $\mathrm{Wu}^{\dagger}$, Xia Zhang ${ }^{\dagger}$, Zhuopeng Wang ${ }^{\dagger}$, Yan $\mathrm{Xu}^{\dagger \dagger}$,*

'Department of Chemistry, College of Sciences, Northeastern University, Shenyang, Liaoning 110819, China

${ }^{\ddagger}$ College of Materials Science and Engineering, Shenyang University of Chemical Technology, Shenyang, Liaoning 110142, China

\footnotetext{
*xuyanjlu@126.com (Yan Xu)
} 


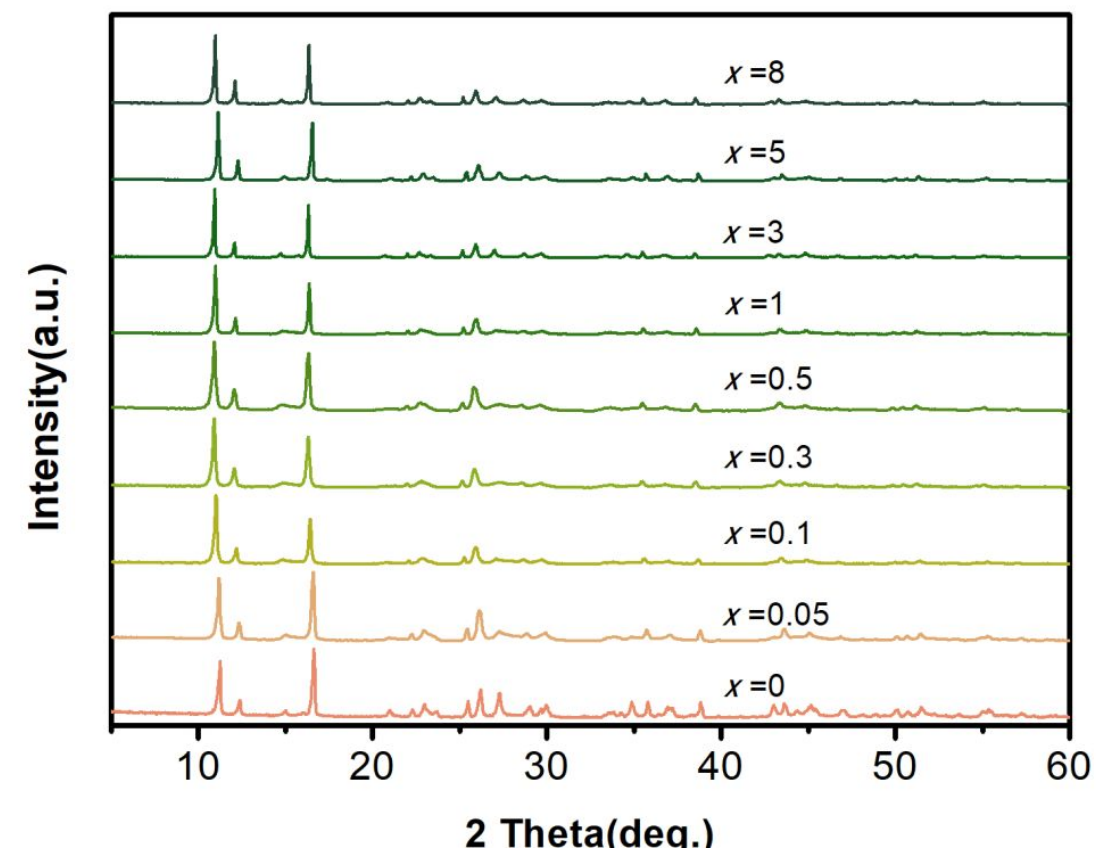

Fig. S1 The experimental XRD patterns of $\operatorname{Er}-\operatorname{MOF}(8,5,3,1,0.5,0.3,0.1,0.05$ and 0 mmol TEAB).
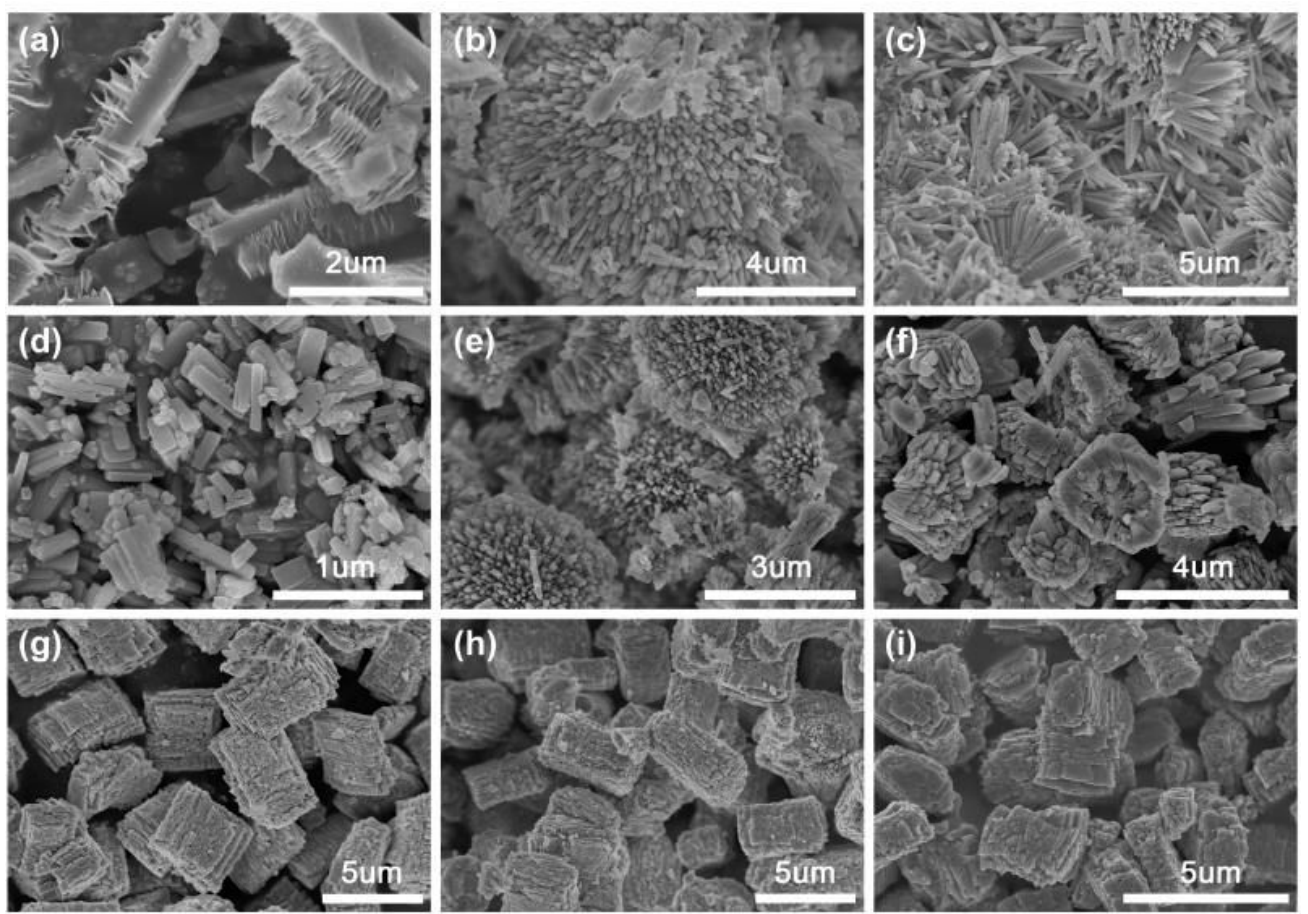

Fig. S2 SEM images of Er-MOF(a, b, c, d, e, f, g and i = 8, 5, 3, 1, 0.5, 0.3, 0.1, 0.05 and 0 mmol TEAB). 


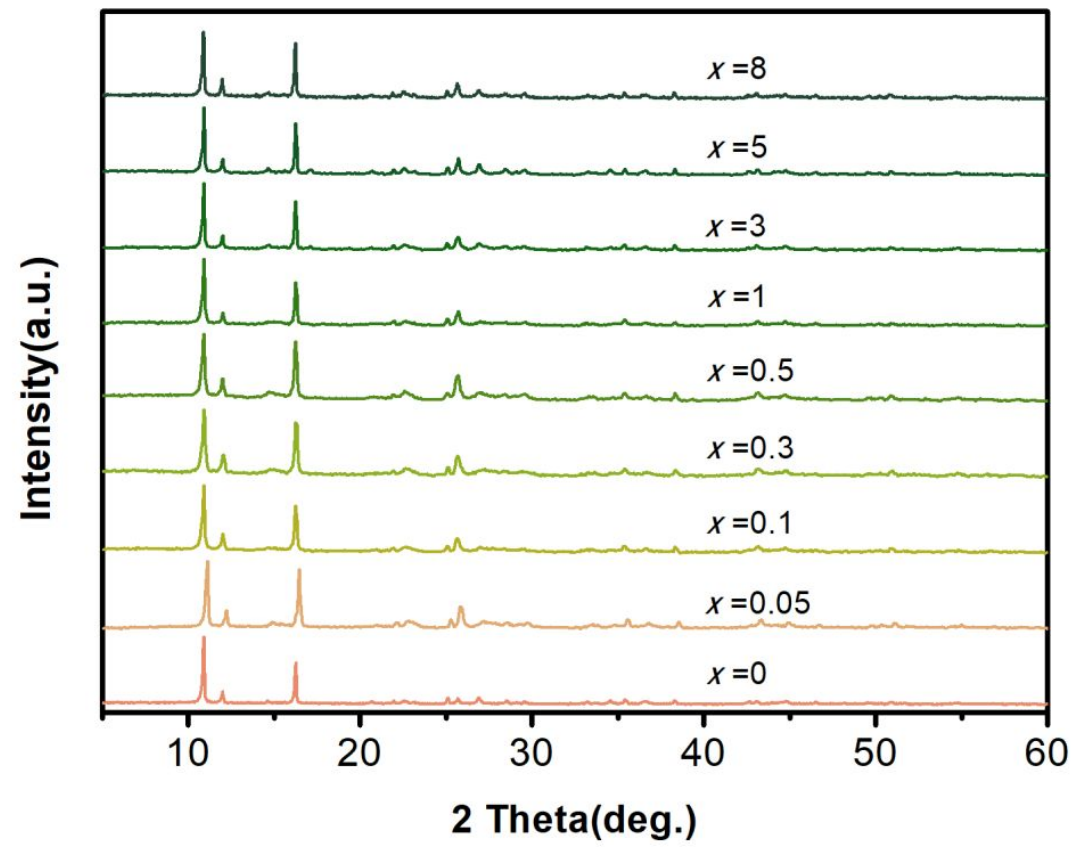

Fig. S3 The experimental XRD patterns of $\operatorname{Dy}-\operatorname{MOF}(8,5,3,1,0.5,0.3,0.1,0.05$ and 0 mmol TEAB).
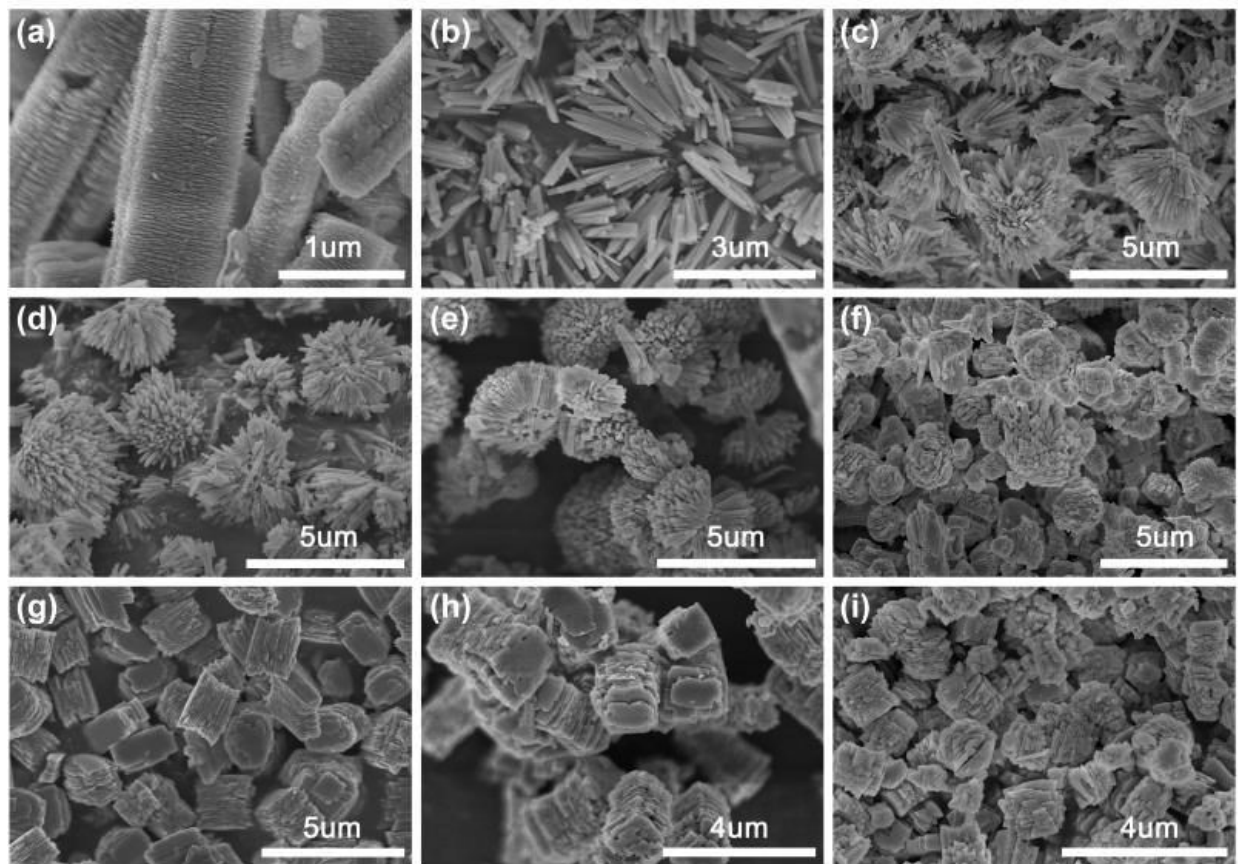

Fig. S4 SEM images of Dy-MOF(a, b, c, d, e, f, g and $i=8,5,3,1,0.5,0.3,0.1,0.05$ and 0 mmol TEAB). 


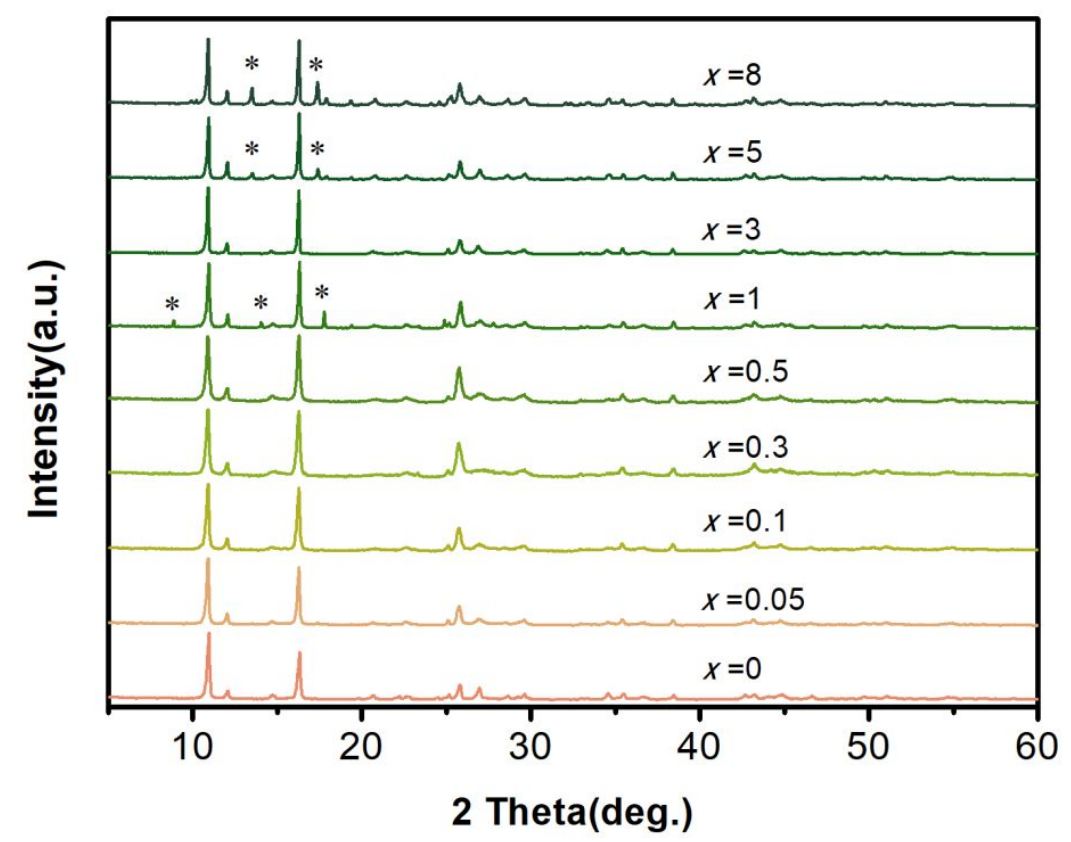

Fig. S5 The experimental XRD patterns of $\mathrm{Y}-\mathrm{MOF}(8,5,3,1,0.5,0.3,0.1,0.05$ and 0 mmol TEAB). The symbol "*" indicates that the peak can be attributed to Eu-BTC (CCDC No. 290771).
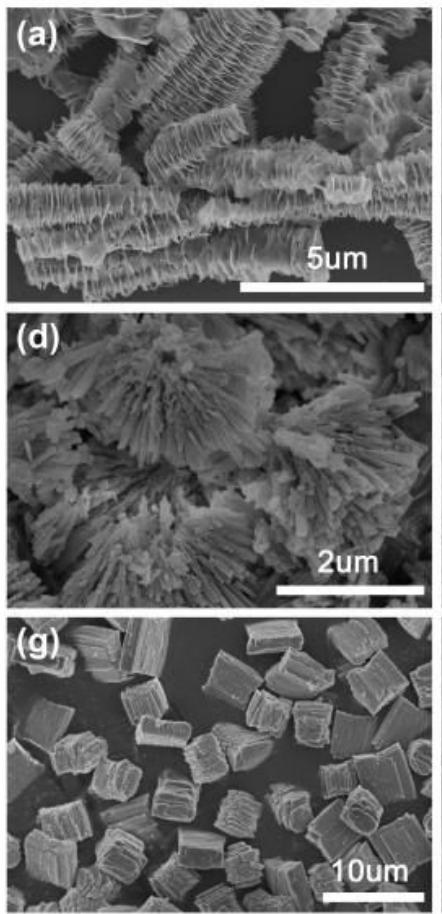
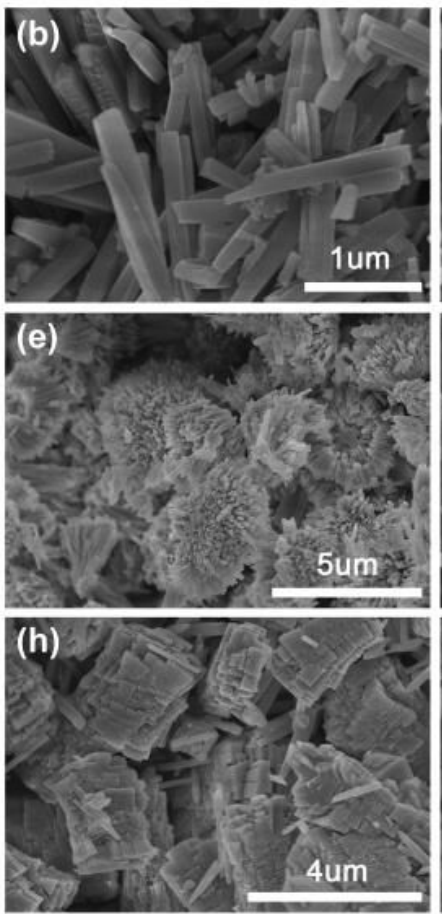
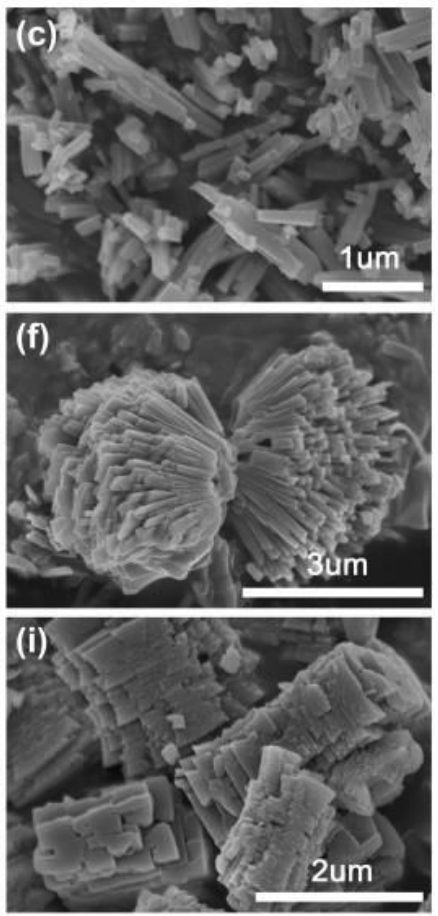

Fig. S6 SEM images of Y-MOF(a, b, c, d, e, f, g and i = 8, 5, 3, 1, 0.5, 0.3, 0.1, 0.05 and 0 mmol TEAB). 


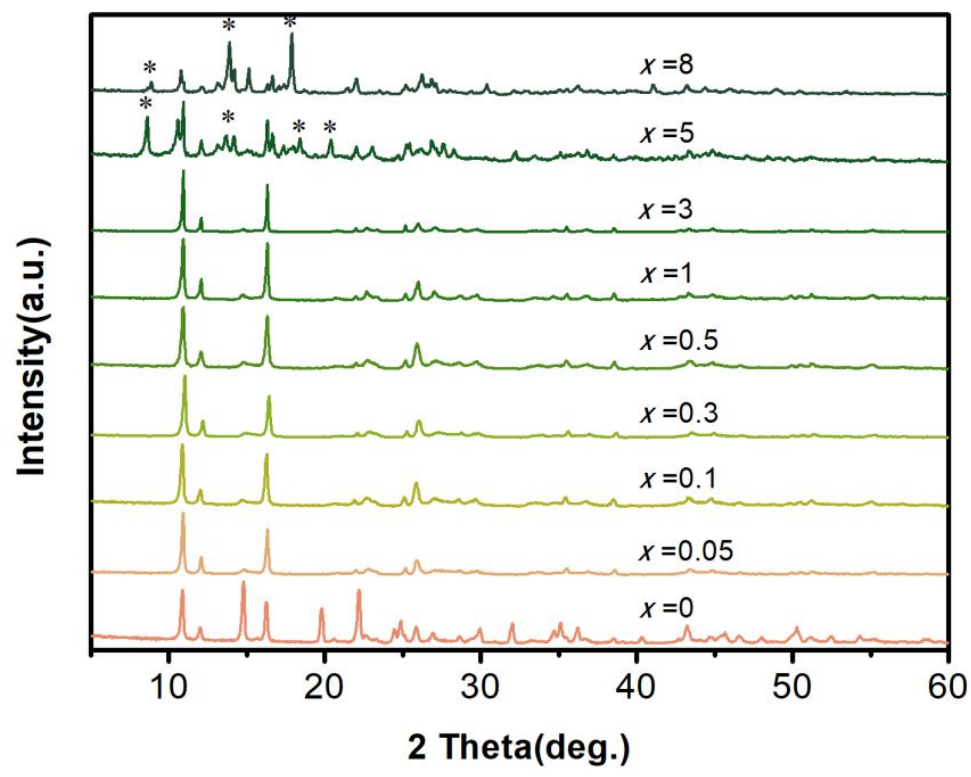

Fig. S7 The experimental XRD patterns of $\operatorname{Tm}-\operatorname{MOF}(8,5,3,1,0.5,0.3,0.1,0.05$ and 0 mmol TEAB). The symbol "*” indicates that the peak can be attributed to Eu-BTC (CCDC No. 290771).
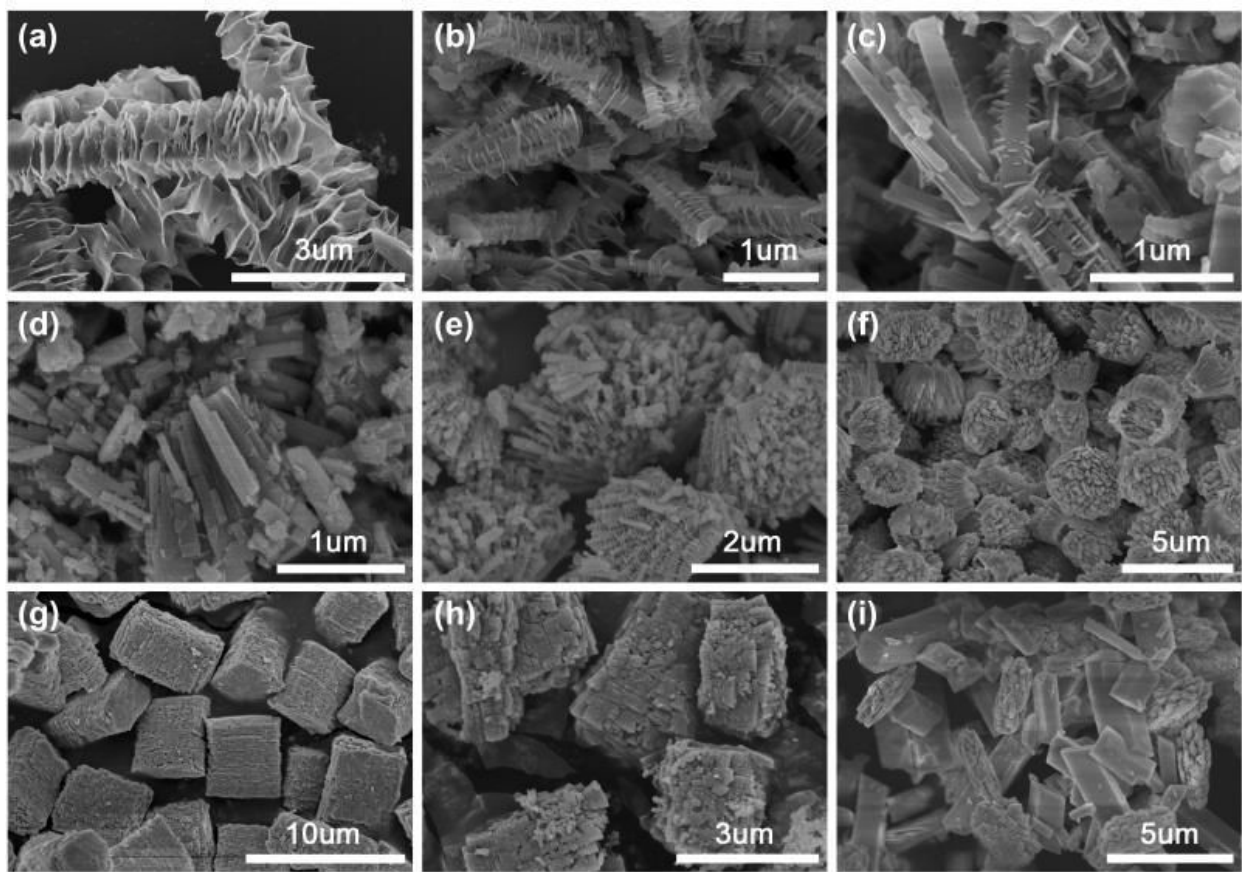

Fig. S8 SEM images of Tm-MOF(a, b, c, d, e, f, g and i = 8, 5, 3, 1, 0.5, 0.3, 0.1, 0.05 and 0 mmol TEAB. 


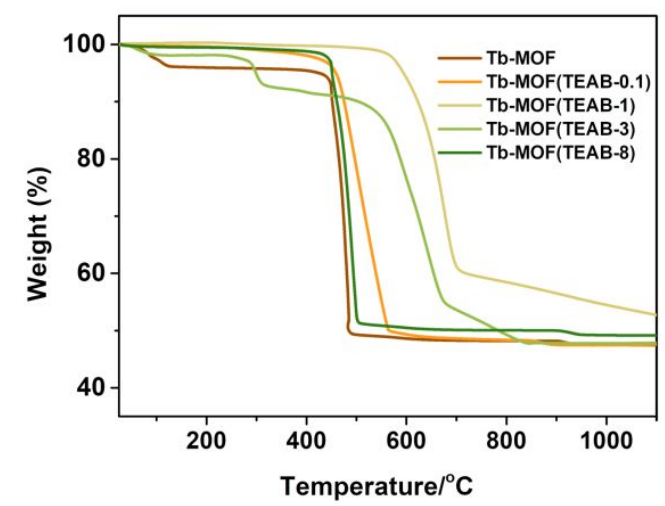

Fig. S9 TG curves of Tb-MOFs prepared from $0,0.1,1,3$ and 8 mmol of TEAB, respectively.
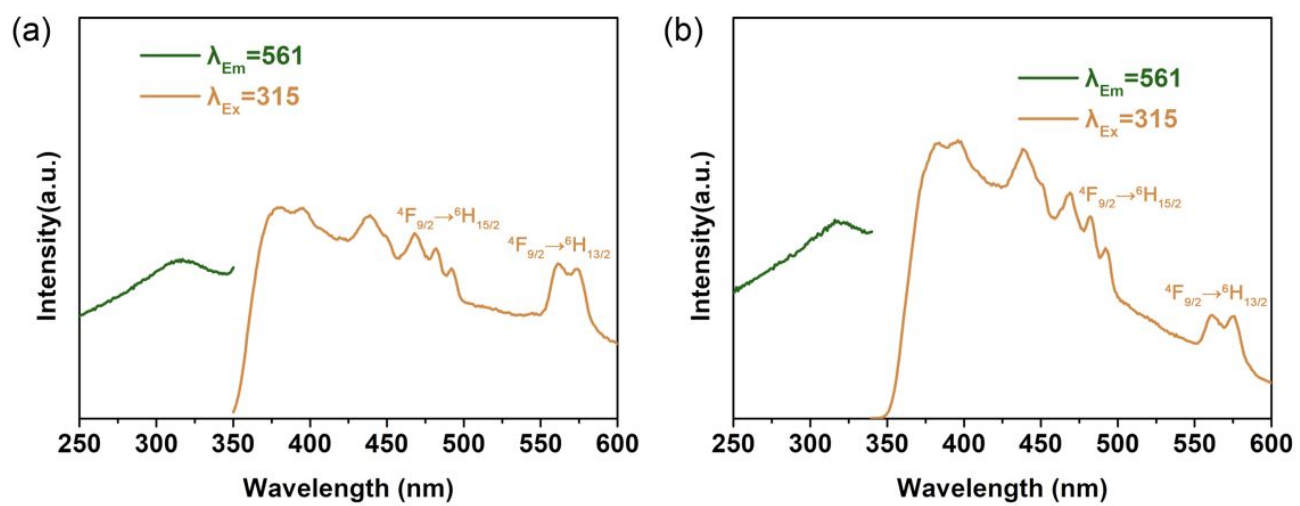

Fig. S10 The photoluminescence excitation and emission spectra of Dy-MOF prepared (a) without the presence of TEAB, and in the presence of 5 mmol TEAB.

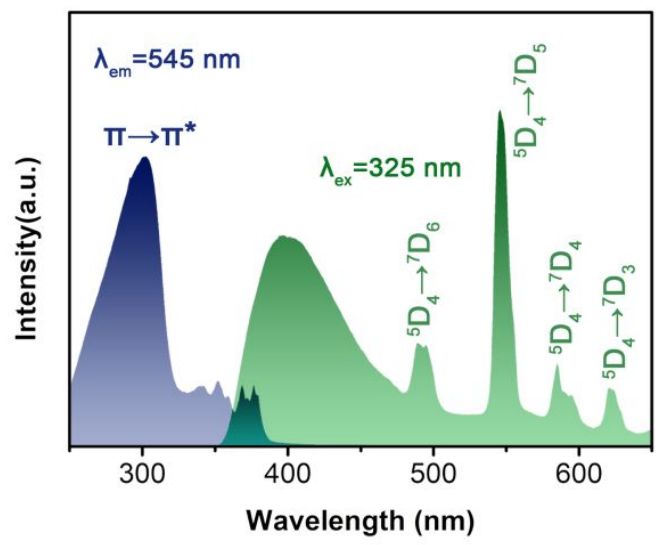

Fig. S11 Photoluminescence excitation and emission spectra of Tb-MOF obtained from 5 mmol TEAB. 
Table S1 The morphological evolution of Eu-MOF, Tb-MOF, Er-MOF, Dy-MOF, Y-MOF, and Tm-MOF by adding different amount of TEAB.

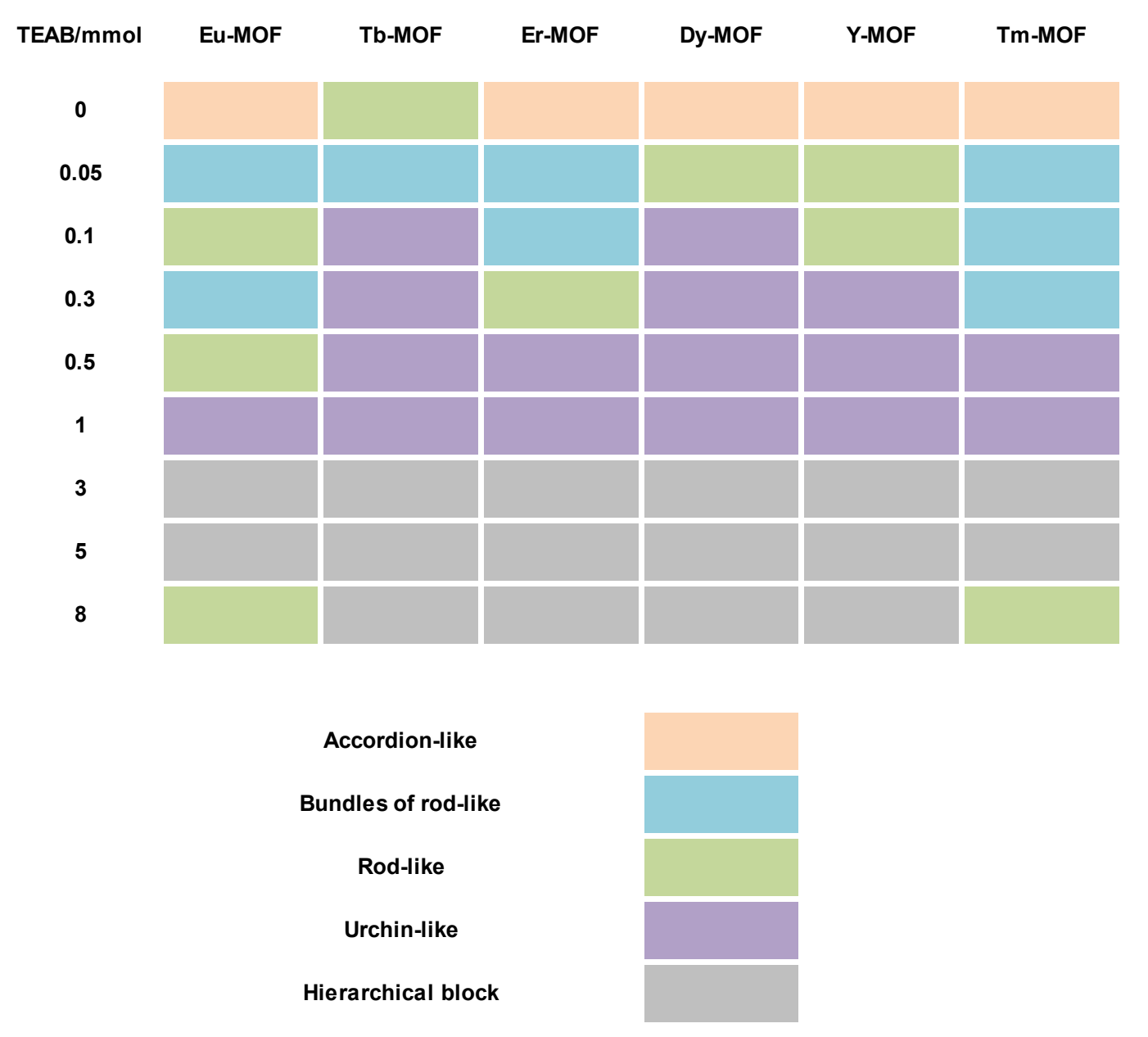

Table S2 Elemental analysis of C, H, N, and O in Eu-MOF, Eu-MOF (TEAB, 0.03 mmol), and Eu-MOF (TEAB, $3 \mathrm{mmol}$ ), respectively.

\begin{tabular}{lllll}
\hline Luminescent materials & $\mathrm{C}(\mathrm{wt} \%)$ & $\mathrm{H}(\mathrm{wt} \%)$ & $\mathrm{N}(\mathrm{wt} \%)$ & $\mathrm{O}(\mathrm{wt} \%)$ \\
\hline Eu-MOF & 30.29 & 1.32 & 0.15 & 21.63 \\
Eu-MOF (TEAB, 0.3 mmol) & 30.24 & 1.33 & 0.35 & 21.71 \\
Eu-MOF (TEAB, 3 mmol) & 30.28 & 3.48 & 2.08 & 23.95 \\
\hline
\end{tabular}

